\title{
Opioid Mortality Following Implementation of Medical Cannabis Programs in the United States
}

\author{
Authors \\ Daniel E. Kaufman ${ }^{1}$, Asawer M. Nihal' ${ }^{1}$, Janan D. Leppoํㅜ, Kelly M. Staples ${ }^{1}$, Kenneth L. McCall², Brian J. Piper ${ }^{1,3}$
}

\author{
Affiliations \\ 1 Geisinger Commonwealth School of Medicine, Scranton, \\ PA, USA \\ 2 University of New England, Portland, ME, USA \\ 3 Center for Pharmacy Innovation and Outcomes, \\ Forty Fort, PA, USA
}

received 21.06 .2020

revised 09.12 .2020

accepted 04.01.2021

published online $\quad 23.2 .2021$

\author{
Bibliography \\ Pharmacopsychiatry 2021; 54: 91-95 \\ DOI 10.1055/a-1353-6509 \\ ISSN $\quad 0176-3679$ \\ (C) 2021. Thieme. All rights reserved. \\ Georg Thieme Verlag KG, Rüdigerstraße 14, \\ 70469 Stuttgart, Germany
}

Correspondence

Brian J. Piper, PhD

525 Pine Street

18509 Scranton

PA

USA

psy391@gmail.com; bpiper@som.geisinger.edu

Supplementary Material is available under https://doi.org/10.1055/a-1353-6509

\begin{abstract}
Introduction The United States is in the midst of an opioid overdose epidemic. Emerging evidence suggests that medical cannabis (MC) may reduce use of opioids for pain in some individuals, with potential impacts on opioid-related overdose. However, there may be other important differences between states that did, and did not, adopt MC.

Methods This study evaluated differences following legal MC sales on US opioid-related overdose deaths, corrected for population, from 1999 to 2017 using an interrupted time series. Comparisons by MC status were also made for Medicaid expansion and the Centers for Disease Control death certificate reporting quality (0: <good, 1 : good, 2 : excellent).

Results Overdose deaths were significantly higher in MC states from 2012-2017. Overdose death slopes over time increased in states with (pre $=1.46 \pm 0.46$, post $=2.90 \pm 0.58$, $p<0.05$ ) and without (pre $=0.20 \pm .10$, post $=1.04 \pm 0.22$, $\mathrm{p}<0.005)$ MC. Post-legalization slopes were significantly higher in MC states $(p<0.01)$. Two states without $(11.1 \%)$ as compared to 11 states with (91.7\%) MC expanded Medicaid by $2014\left(X^{2}[1]=19.03, p<0.0005\right)$. MC states $(1.50 \pm 0.23)$ had higher death certificate reporting quality relative to states without MC $(0.78 \pm 0.22, \mathrm{p}<0.05)$.

Discussion $\mathrm{MC}$ states had higher rates of opioid overdoses. Although there was no decrease in association with MC introduction, these results were confounded by states without MC having lower overdose reporting quality. Medicaid expansion was also more common in states with MC. Finally, the potency of fentanyl analogues may have obscured any protective effects of MC against illicit opioid harms.
\end{abstract}

\section{Introduction}

The United States (US) continues to struggle to address an opioid overdose crisis. There are 5 to 8 million Americans who use prescription opioids for chronic pain each year [1] relative to 17000 overdoses that involved a prescription opioid [2]. The year 2018 may have had fewer drug overdoses nationally than 2017, although Delaware and Missouri had appreciable (>15\%) increases [3]. Overdose death data in the US should be interpreted cautiously, as states differ widely in use of medically and non-medically trained personnel and analytical chemistry procedures to determine cause of death and substances involved. The number of overdoses from 1999 to 2015 where the substance involved was unspecified on the death certificate ranged from $0 \%$ in Washington DC to 51 \% in Pennsylvania [4]. The US Centers for Disease Control and Prevention (CDC) uses a 3-tier system (excellent, good, or other/less than good) to classify state reporting of overdose fatality [5]. Over twofifths (44\%) of states fall into the latter designation ( $>$ Fig. 1). Prescription opioid use, a measure with much more homogenous data collection, peaked in 2011 and has undergone pronounced reductions for most agents [6], with the exception of buprenorphine [7]. Buprenorphine is an opioid partial agonist, and it is often formulated with the opioid antagonist naloxone. Buprenorphine availability is associated with decreased opioid overdoses [8]. States that 


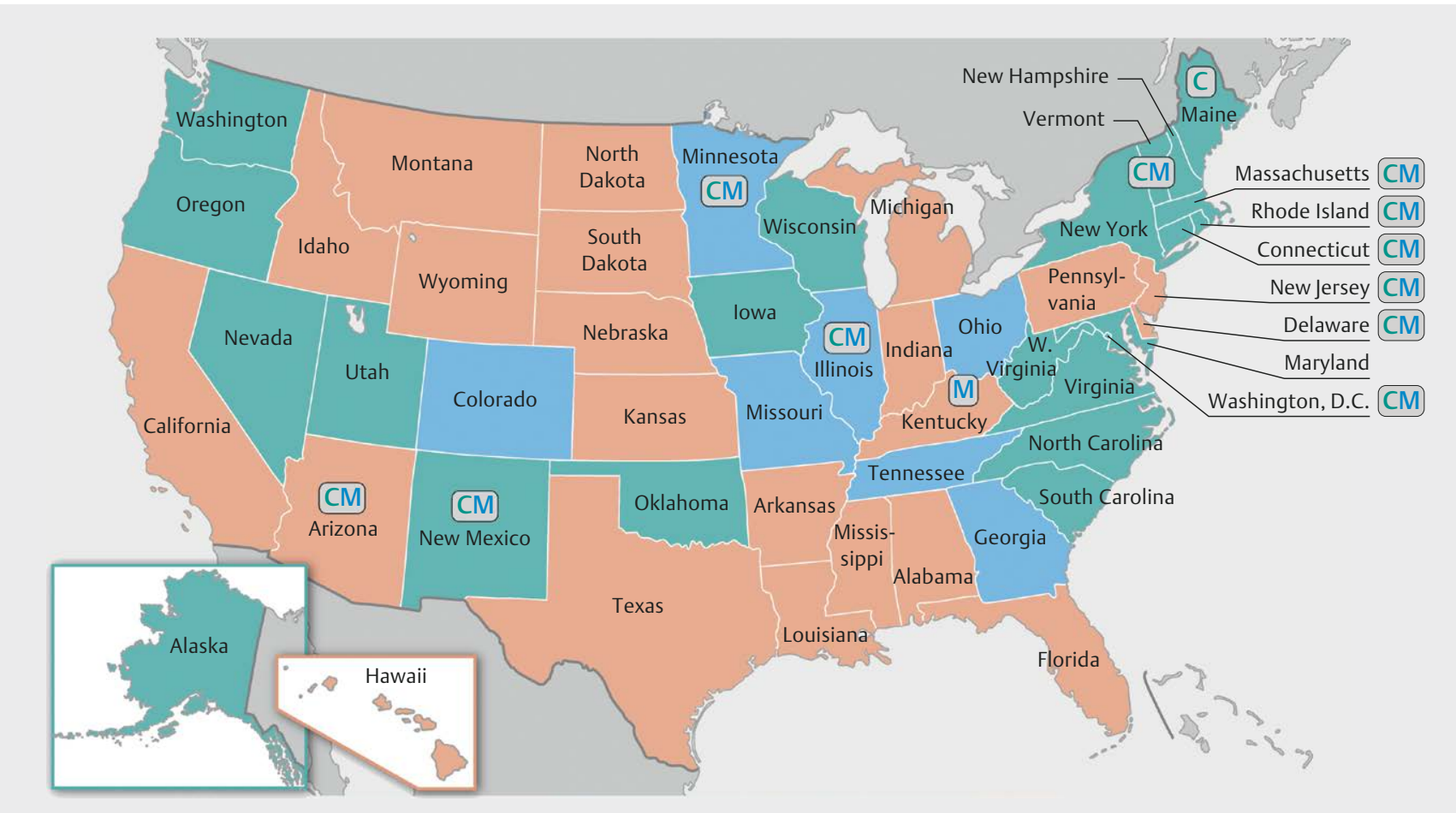

- Fig. 1 Heat map of the US by overdose death certificate reporting quality [5]. Excellent (green) was defined as $\geq 90 \%$ of drug overdose death certificates mentioning at least 1 specific drug in 2014, with the change in percentage of drug overdose deaths mentioning at least 1 specific drug differing by $<10$ percentage points from 2014 to 2015 . Good (blue) was defined as $80 \%$ to $<90 \%$ of drug overdose death certificates mentioning $\geq 1$ specific drug in 2014 , with the change in the percentage of drug overdose deaths mentioning $\geq 1$ specific drug differing by $<10$ percentage points from 2014 to 2015. The remaining states were classified as less than good (pink). M: Medicaid expansion by 2014. C: medical cannabis state.

expanded Medicaid saw a $70 \%$ increase in buprenorphine prescriptions [9].

Several lines of evidence, albeit mostly from non-randomized and non-blind research designs, are suggestive of the potential for medical cannabis (MC) to attenuate opioid use/misuse. The potency of morphine on the rodent tail flick response to an aversive stimuli was greatly enhanced by tetrahydrocannabinol (THC), which indicates that less morphine would be needed to reduce pain [10]. Similarly, human trials have supported the ability of THC to augment the pain-reducing effects of morphine and oxycodone [11]. Three-quarters of MC dispensary members reported a reduction in their use of opioids after starting MC [12]. Similarly, examination of a prescription drug monitoring program records revealed that patients were 17-fold more likely to stop use of all controlled substances after starting MC [13]. States that legalized MC had lower expenditures for prescription medications in Medicare [14]. A National Academy of Sciences report summarizing the evidence concluded that cannabis reduces chronic pain in adults, although the magnitude of effect was modest [15].

However, evidence of an association between MC and opioidrelated harm is mixed. In one highly cited study of opioid analgesic and heroin overdoses from 1999-2010, 2 discoveries were made. First, states that legalized MC had more analgesic opioid overdoses relative to those that did not. Second, opioid overdoses declined following MC implementation relative to those states without MC [16]. A later replication and extension of that work found that the association between MC and opioid-related overdose death reversed over time, so that, by 2017, MC laws were associated with an increase in overdose deaths [17]. Pre-existing state differences were found in opioid dependence hospitalizations but also reductions associated with MC implementation [18]. Findings like these may have been influential for 8 US states (Colorado, Illinois, Missouri, Nevada, New Jersey, New Mexico, New York, and Pennsylvania) including opioid misuse as a qualifying condition for MC.

The objective of this report was to provide additional data regarding the association between opioid overdose mortality and MC program implementation. This extends upon and clarifies the discrepant outcomes reported previously $[16,17,19]$.

\section{Methods}

\section{Procedures}

The opioid overdose mortality rate from 1999-2017 in each state was extracted from the Centers for Disease Control and Prevention's Wide-ranging Online Data for Epidemiologic Research (WONDER) database [20], which draws from death certificates for US residents. We coded these data broadly and narrowly. Opioid overdose deaths were broadly defined using the International Statistical Classification of Diseases, $10^{\text {th }}$ revision codes: X40-44, X60-64, $X 85, Y 10-Y 14[16]$. Only data that was coded for illicit and prescription opioids (T40.0-T40.4) was used. This included opium, heroin, 
other opioids, methadone, and other synthetic opioids. The narrow definition was more restrictive and excluded illicit opioids (T40.0 opium and T40.1 heroin) and X85 (assault by drugs).

An interrupted time series examined trends around the time of states' MC program implementation. We defined the start dates of MC programs as the year MC sales first began in the state. These data were found on either the state's MC program website and from publications detailing the start of MC sales (Supplemental > Table. 1). Only Arizona (implemented in 2012), Connecticut (2014), Delaware (2015), District of Columbia (2013), Illinois (2015), Maine (2011), Massachusetts (2015), Minnesota (2015), New Jersey (2012), New Mexico (2009), Rhode Island (2013), and Vermont (2013) had start dates within the year range of 1999-2017, with 3 years of data available both pre- and post-MC program implementation. Secondary analyses were completed based on earlier law enactment and effect dates [21]. The states without medical cannabis laws (Alabama, Georgia, Idaho, Indiana, Iowa, Kansas, Kentucky, Louisiana, Mississippi, Nebraska, North Carolina, South Carolina, South Dakota, Tennessee, Texas, Virginia, Wisconsin, and Wyoming), irrespective of cannabidiol only laws, served as a comparison group. The research was deemed exempt by the IRB of the University of New England.

\section{Data analysis}

Statistical analysis was conducted using Systat software, v13.1. First, opioid overdoses were compared over 19 years for states with versus without MC ( + versus - ). If the assumption of homogeneity of variance was not met $(p<0.10)$, a separate variance t-test was completed. Second, an interrupted time series was completed. Slopes for opioid overdoses from 3 years before the implementation of medical cannabis (pre-), and 3 years after (post-)-the year of implementation was excluded as a transitional year-were calculated for MC + and MC - states with GraphPad Prism 8. The mean MC program implementation date (2012) was used as the interruption point for the comparison group. Comparisons were made during the pre- and post-periods. Third, slopes from 1999 to 2017 (i.e., a non-interrupted time series) were calculated and MC + versus MC - states compared.

Two potential confounds were also examined. A 2 (MC - vs. $M C+$ ) by 2 (Medicaid expansion by $1 / 1 / 2014$ or $12 / 2017$ ) chisquare and a t-test on the CDC's 3-tiered classification system [5] for the quality of death certificate reporting ( 2 = very good/excellent, 1 = good, or 0 = less than good based on completeness and consistency over time) were completed. Variability was expressed as the SEM. A p-value less than 0.05 was considered statistically significant.

\section{Results}

Opioid overdoses, broadly defined, had pronounced elevations over time and were generally higher among MC + states. Significant differences in overdoses per 100,000 population were identified in '02, '06, and ' 13 to ' 17 between MC + and MC - states (> Fig. 2a).

- Figure $\mathbf{2 b}$ illustrates that the linear-regression slope of opioid overdose over time. The slope was significantly larger among states that went on to implement MC during the pre-MC period $(t[13.07]=3.16, p<0.01)$. Slopes increased significantly in states without $M C(t[17]=3.57, p<0.005)$. The change in states with $M C$ was also significant $(t[11]=2.45, p<0.05)$. The post-legalization slope was significantly higher in $M C+$ than $M C-(t[14.35]=3.01$, $\mathrm{p}<0.01)$ states. Additional analyses based on the date of MC law enactment and the effective date are in Supplemental $>$ Table 2. During the 1999 until 2017 period, overdose slopes were significantly greater in $\mathrm{MC}$ states $(0.876 \pm 0.127)$ relative to those that did not enact $M C$ laws $(0.425 \pm 0.068, t[28]=3.40, p<0.005)$.

Two MC - (11.1\%) as compared to eleven MC+ $(91.7 \%)$ states expanded Medicaid by $2014\left(X^{2}[1]=19.03, p<0.0005\right)$. The same general pattern was noted when the expansion date was set at 2017 $\left(M C-=22.2 \%, M C+=91.7 \%, X^{2}[1]=13.89, p<0.0005\right)$. The quality of overdose determinations was twice as high in $\mathrm{MC}+$ states $(t[28]=2.18, p<0.05$, $>$ Fig. $2 c)$. Irrespective of $M C$, states rated excellent, relative to those that were less than good, had significantly more reported overdoses in 2013-2017 (Supplemental Fig. 1a).

Additional analyses were completed on opioid overdoses narrowly defined to exclude heroin/opium. Overdoses per 100000 were significantly $(p \leq 0.02)$ higher in $M C+$ relative to $M C$ - in 2015, 2016, and 2017 (Supplemental $>$ Fig. 2a). The slopes were not significantly larger in $\mathrm{MC}+(+0.79 \pm 0.42)$ compared to $\mathrm{MC}-$ states $(-0.10 \pm 0.09, t[10.86]=2.05, p=0.066)$ before legalization. This difference was significant after legalization $(M C+=3.17 \pm 0.71$, MC $-=0.80 \pm 0.21, t[12.97]=3.20, p<0.01$, Supplemental $>$ Fig. 2b). States with excellent overdose death reporting, independent of MC policies, reported more overdoses from 2013 to 2017 than states whose reporting was less than good (Supplemental > Fig. 1b).

\section{Discussion}

This reports provides a key addition to the prior literature evaluating the potential population health benefits in the US of MC $[16,17]$. Previous work has found that states with MC from 19992010 had elevated opioid mortality, relative to those without MC [16]. We observed that this trend held with the inclusion of 7 additional years of data ( $\triangleright$ Fig. 2a). However, we found that, contrary to this previous work [16], opioid overdoses did not decrease in the years subsequent to states adopting MC as compared to states that did not. In fact, states that adopted MC had significantly greater overdose slopes than those that did not ( $>$ Fig. 2b). This finding is similar to that of [17], which found that the association between MC laws and opioid-related overdose reversed over time so that, by 2017, MC laws were associated with significant increases in opioid overdose fatality. The preclinical [10] and epidemiological evidence base [12-15] indicates that it is plausible that MC could decrease opioid use with its synergistic anti-nociceptive properties [11] and therefore limit misuse and overdose potential. However, most work in this area assumes that overdose determinations are consistently made by medical examiners and coroners across the US ( $\triangleright$ Fig. 1). At the very least, any differences should be random and not systematically different based on MC laws. This assumption was not warranted ( $>$ Fig. $\mathbf{2 c}$ ) in that states without MC had lower overdose death determination reporting quality using the CDC criteria [5]. The most parsimonious interpretation of the present data, and others [17], is that attempting to conduct ecologi- 


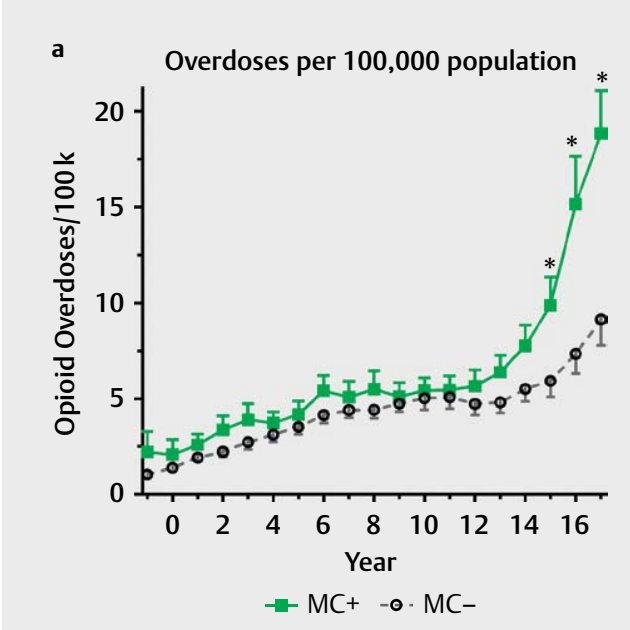

b Opioid overdose slope
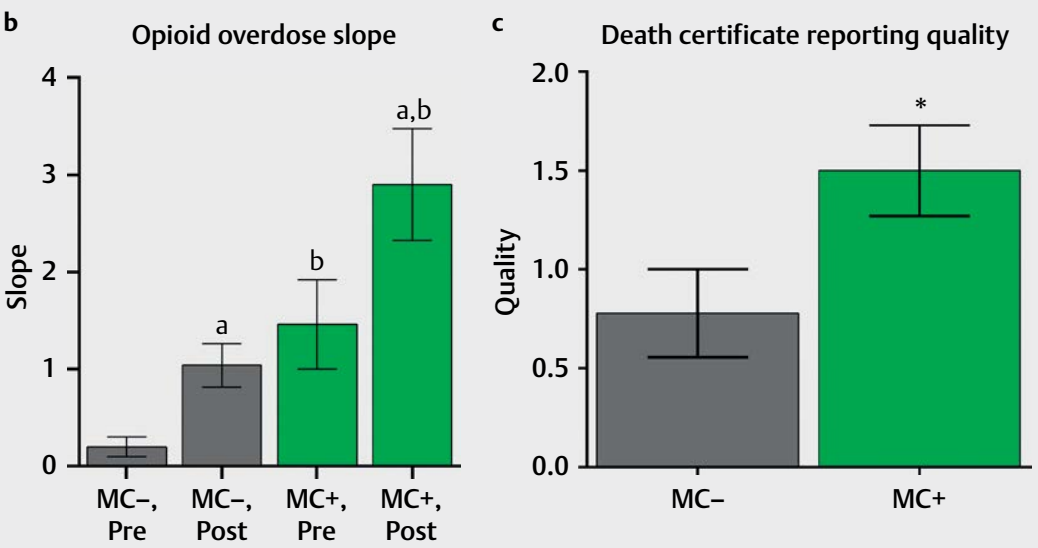

-Fig. 2 Opioid overdoses in the US as reported by the CDC's Wide-ranging Online Data for Epidemiologic Research from 1999 to 2017 as a function of presence $(+)$ or absence $(-)$ of state medical cannabis (MC) law (a, ${ }^{*} \mathrm{p}<0.05$ versus $\mathrm{MC}-$ ). Opioid overdose slopes 3 years before (pre) and 3 years after (post) state MC implementation (b, a $\mathrm{p}<0.05$ versus pre; ${ }^{\mathrm{b}} \mathrm{p}<0.05$ versus corresponding $\mathrm{MC}-$ ). Death certification reporting quality $(\mathbf{c}$, excellent: 2 , good: 1 , less than good: $0 ;{ }^{*} p<0.05$ ).

cal level studies to determine whether MC limits opioid overdoses is a non-optimal research design due to the many confounds that are present at a state level that differentiate MC and non-MC states.

These findings should not be used to discount that the risk-tobenefit ratio may still favor MC as a tool that may be endorsed by some chronic pain patients, especially relative to the overdose potential of prescription opioids. As others have described in some detail [17], the ecological research design where the unit of analysis is the state does not adequately address this question. A potential follow-up study would be to examine individual OUD patients in states where $\mathrm{MC}$ is a qualifying condition for OUD. State-level MC systems, either established through voter initiative or legislation, may differ on a wide-variety of socio-political characteristics. Additional research could use multiple regression to account for multiple confounders. Medicaid expansion was much more common among MC + states. The additional resources provided by Medicaid could have provided for additional buprenorphine [7, 9] which initially decreased opioid overdoses [8]. Later, the potency of fentanyl and many fentanyl analogues [22] could overcome any cannabis substitution effect [12]. The fact that fentanyl-related overdoses, which have driven much of the increase in opioid-related deaths over the past several years are concentrated in the Eastern US, may be a significant confounder because the majority (83.3\%) of the MC+ states were east of the Mississippi River.

Some caveats and future areas of study should be noted. The majority of long-term prescription opioid users neither misuse those medications nor experience an overdose [23]. This report was based on US overdoses as reported by the CDC. Five states had more than one-third of their death certificates where the substance involved was unspecified [4]. The non-uniformity of autopsy procedures (e.g., When did each state, county, or city start testing for fentanyl? Were screens or the more expensive confirmatory testing employed? How many of the 200 fentanyl analogues were tested in each municipality and when?) is a substantial challenge to re- search and a barrier for empirically informed public policy that utilizes this information. Although this would limit the power to detect differences, future research might consider focusing only on the subset of areas where the death determination procedures have been consistently high [22]. Additional qualitative studies [24] could examine patient views on prescription or illicit opioid use after starting $\mathrm{MC}$ and provide insight into the many confounds inherent to this topic (e.g., state level differences in naloxone distribution). This report did not adjust for multiple statistical comparisons although analyses that met more conservative thresholds (e.g., $\mathrm{p}<0.01$ ) were noted. This study investigated MC only and may not generalize to cannabidiol or recreational cannabis laws.

In conclusion, new empirically grounded solutions to reverse the pronounced levels of opioid overdoses in the US are urgently needed. This study tested whether the protective effects previously found in some studies of MC against opioid overdoses could be repeated with the addition of more data. States with MC had increased, not decreased as would be predicted, overdose slopes. An impediment to empirically informed public policy is that overdose determinations procedures are not regionally or temporally uniform. This study determined that states with MC had significantly higher quality of death certificate reporting. Additional investigations using other research designs and dependent measures are ongoing to further our understanding of the population health benefits and risks (e.g., automobile accidents) produced by the increased availability of medical and recreational cannabis in the US and internationally.

\section{Acknowledgements}

Thank you to Iris Johnston for technical assistance. 


\section{Funding}

Prior research has been supported by NIDA and the Center for Wellness Leadership, travel by cannabis patient organizations (e.g. Patients Out of Time). Current research on osteoarthritis is supported by Pfizer and Eli Lilly.

\section{Conflict of Interest}

BJP has received research support from the medical marijuana dispensaries and travel from cannabis advocacy non-profit organizations (e.g. Patients Out of Time). He is part of an osteoarthritis research team supported by Pfizer and Eli Lilly. The other authors have no disclosures.

\section{References}

[1] Reuben DB, Alvanzo AA, Ashikaga T et al. National Institutes of Health pathways to prevention workshop: the role of opioids in the treatment of chronic pain. Ann Intern Med 2015; 162: 295-300. doi: 10.7326/ M14-2775

[2] Seth P, Scholl L, Rudd RA et al. Deaths involving opioids, cocaine, and psychostimulants - United States, 2015-2016. MMWR Morb Mortal Wkly Rep 2018; 67: 349-358. doi: 10.15585/mmwr.mm6712a1

[3] Ahmad FB, Escobedo LA, Rossen LM et al. Provisional drug overdose death counts. National Center for Health Statistics. 2019; accessed 3/26/20 at https://www.cdc.gov/nchs/nvss/vsrr/drug-overdose-data. htm

[4] Buchanich JM, Balmert LC, Williams KE et al. The effect of incomplete death certificates on estimates of unintentional opioid-related overdose deaths in the United States, 1999-2015. Public Health Rep 2018; 133: 423-431. doi: 10.1177/0033354918774330

[5] Rudd RA, Seth P, David F et al. Increases in drug and opioid-involved overdose deaths - United States, 2010-2015. MMWR Morb Mortal Wkly Rep 2016; 65: 1445-1452. doi: 10.15585/mmwr.mm655051e1

[6] Piper B], Shah DT, Simoyan OM et al. Trends in medical use of opioids in the U.S., 2006-2016. Am J Prev Med 2018; 54: 652-660. doi: 10.1016/j.amepre.2018.01.034

[7] Pashmineh Azar AR, Cruz-Mullane A, Podd JC et al. Rise and regional disparities in buprenorphine utilization in the United States. Pharmacoepidiol Drug Saf. 2020; doi: 10.1002/pds.4984

[8] Schwartz RP, Grycznski J, O'Grady KE et al. Opioid agonist treatments and heroin overdose deaths in Baltimore, Maryland, 1995-2009. Am J Pub Health 2013; 103: 917-922. doi: 10.2105/AJPH.2012.301049

[9] Wen H, Hockenberry JM, Borders TF et al. Impact of Medicaid expansion on Medicaid-covered utilization of buprenorphine for opioid use disorder treatment. Med Care 2017; 55: 336-341. doi: 10.1097/ MLR.0000000000000703
[10] Smith FL, Cichewicz D, Martin ZL et al. The enhancement of morphine antinociception in mice by delta9-tetrahydrocannabinol. Pharmacol Biochem Behav 1998; 60: 559-566 PMID: 9632241

[11] Abrams DI, Couey P, Shade SB et al. Cannabinoid-opioid interaction in chronic pain. Clin Pharmacol Ther 2011; 90: 844-851. doi: 10.1038/ clpt.2011.188

[12] Piper B], DeKeuster RM, Beals ML et al. Substitution of medical cannabis for pharmaceutical agents for pain, anxiety, and sleep. J Psychopharmacology 2017; 31: 569-575. doi: $10.1177 / 0269881117699616$

[13] Stith SS, Vigil JM, Adams IM et al. Effects of legal access to cannabis on scheduled II-V drug prescriptions. I Am Med Dir Assoc 2018; 19 : 59-64.e1. doi: 10.1016/j.jamda.2017.07.017

[14] Bradford AC, Bradford WD. Medical marijuana laws reduce prescription medication use in Medicare Part D. Health Affairs 2016; 35: 1230 1236. doi: $10.1377 /$ hlthaff.2015.1661

[15] National Academies of Sciences, Engineering, and Medicine. The Health Effects of Cannabis and Cannabinoids: The Current State of Evidence .and Recommendations for Research. Washington, DC: The National Academies Press; 2017

[16] Bachhuber MA, Saloner B, Cunningham CO et al. Medical cannabis laws and opioid analgesic overdose mortality in the United States, 1999-2010. JAMA Intern Med 2014; 174: 1875. doi: 10.1001/ jamainternmed.2014.4005

[17] Shover CL, Davis CS, Gordon SC et al. Association between medical cannabis and opioid overdose mortality has reversed. Proc Natl Acad Sci USA 2019; 116: 12624-12626. doi: 10.1073/pnas.1903434116

[18] Shi Y. Medical marijuana policies and hospitalizations related to marijuana and opioid pain reliever. Drug Alcohol Depend 2017; 173: 144-150. doi: 10.1016/j.drugalcdep.2017.01.006

[19] Powell D, Pacula RL, Jacobson M.. Do medical marijuana laws reduce addictions and deaths related to pain killers? J Health Econ 2018; 58: 29-42

[20] Centers for Disease Control and Prevention. CDC WONDER; Accessed 3/26/20 at https://wonder.cdc.gov/

[21] Bradford AC, Bradford WD. Medical marijuana laws may be associated with a decline in the number of prescriptions for Medicaid enrollees. Health Aff 2017; 36: 945-951. doi: 10.1377/hlthaff.2016.1135

[22] Simpson KJ, Moran MT, Foster ML et al. Descriptive, observational study of pharmaceutical and non-pharmaceutical arrests, use, and overdoses in Maine. BM] Open 2019; 9: e027117. doi: 10.1136/ bmjopen-2018-027117

[23] Kroenke K, Cheville A. Management of chronic pain in the aftermath of the opioid backlash. JAMA 2017; 317: 2365-2366

[24] Mercurio A, Aston ER, Claborn KR et al. Marijuana as a substitute for prescription medications: A qualitative study. Subst Use Misuse 2019; 54: 1894-1902. doi: 10.1080/10826084.2019.1618336 